\title{
Big Data com Learning Analytics para apoiar o Planejamento Pedagógico Acadêmico
}

\author{
Sérgio Piter Nogueira ${ }^{1}$, Rodrigo E. Francisco ${ }^{1,2}$, Luciane de F. Silva ${ }^{1,4}$, \\ Márcia A. Fernandes ${ }^{1}$, Cleon X. Pereira Júnior ${ }^{1,3}$ \\ ${ }^{1}$ Faculdade de Computação - Universidade Federal de Uberlândia (UFU) \\ Uberlândia - MG - Brasil \\ ${ }^{2}$ Instituto Federal Goiano (IF Goiano) Campos Belos - GO - Brasil \\ ${ }^{3}$ Instituto Federal Goiano (IF Goiano) Morrinhos - GO - Brasil \\ ${ }^{4}$ Instituto de Informática - Centro Universitário do Triângulo (UNITRI) \\ Uberlândia - MG - Brasil \\ \{sergiopitern, lucianefatsilva\}@gmail.com \\ \{rodrigo.francisco, cleon.junior\}@ifgoiano.edu.br \\ \{marcia\} @ufu.br
}

\begin{abstract}
The use of technologies, such as Virtual Learning Environments and Educational Information Systems in higher education generated structured and unstructured data spread across different bases. The proposal intends to present is, considering these conditions, how to use Learning Analytics (LA) techniques to generate useful information for students, teachers and academic management. The proposal includes an architecture designed to store the data from the different sources in a Big Data and its use as an input to LA. We therefore direct you to a possible solution in with the call of the 7th DesafIE in the period of 10 years, involving the subjects information retrieval, LA and data analysis in educational contents. online
\end{abstract}

Resumo. O uso de tecnologias, como Ambientes Virtuais de Aprendizagem e Sistemas de Informações Educacionais, no ensino superior gerou dados estruturados e não estruturados espalhados em diferentes bases. A proposta que se pretende apresentar é, considerando essas condições, como usar técnicas de Learning Analytics (LA) para gerar informações úteis para estudantes, professores e gestão acadêmica. A proposta inclui uma arquitetura que visa armazenar os dados das diferentes fontes em um Big Data e seu uso como insumo para LA. Assim, trazemos direcionamentos para uma possível solução em conformidade com a chamada do $7^{\circ}$ DesafIE no período de 10 anos, envolvendo os temas recuperação da informação, LA e análise de dados em conteúdos educacionais online.

\section{Introdução}

A ciência vem realizando avanços importantes a partir da experiência de aplicar Inteligência Artificial (IA) e Mineração de Dados em grandes bases de dados. Tais avanços 
têm impactado também no aspecto social, trazendo novas profissões ligadas à Big Data e Data Science.

$\mathrm{Na}$ área de educação, as recentes inovações vêm sendo possibilitadas pelos avanços científicos e tecnológicos da ciência da computação. Conforme [Krikun 2017], o aumento do uso das tecnologias web tem gerado grande massa de dados em diversos domínios e contextos, o que tornou crucial a aplicação da mineração de dados e outras técnicas para extração de informações importantes.

$\mathrm{O}$ advento da internet e a interação em sistemas colaborativos possibilitou a emersão de novas ferramentas para uso na educação, que integram muitos serviços de gerenciamento da aprendizagem, como Moodle ${ }^{1}$, Sakai ${ }^{2}$ e Desire2Learn ${ }^{3}$ [Martins and Giraffa 2015]. O Openredu ${ }^{4}$, e.g., é um Ambiente Virtual de Aprendizagem (AVA) que contém funcionalidades de organização de materiais comuns em sistema de gestão de aprendizagem e inclui, também, aspectos de rede social. O projeto foi concebido visando à simplicidade da experiência de usuários, permitindo que o professor se aproxime da cultura das gerações mais jovens por meio de um canal de comunicação produtivo e com uma proposta mais divertida [Gomes et al. 2017].

Tais ferramentas, além de colaborarem com ensino à distância, viabilizam outra modalidade de aprendizagem que é considerada híbrida, o blended learning ou $b$ learning. Essa modalidade visa à combinação de práticas pedagógicas do ensino presencial e do ensino a distância, colaborando dessa forma em melhorar o desempenho dos estudantes tanto no presencial quanto a distância.

Os AVA's se tornaram ferramentas com alta capacidade de geração de dados educacionais. A análise destes dados tem crescido nos últimos anos, impulsionando uma linha de investigação conhecida como análise de aprendizagem ou Learning Analytics (LA). A LA [Chatti et al. 2014] é composta por grandes áreas tais como pedagogia, psicologia, ciência de dados, web semântica, dentre outras, e visa coletar e analisar dados de estudantes e seus contextos na intenção de verificar a aprendizagem e os ambientes que estão interagindo para que ela ocorra.

O principal objetivo da LA está diretamente relacionado à criação de informações estratégicas e benéficas para a tomada de decisões orientada e pautadas por dados estatísticos. Uma das maneiras de se conseguir este objetivo é utilizar Big Data, com dados de várias fontes. O maior desafio nesta abordagem é determinar como os dados serão capturados, padronizados, processados, armazenados, apresentados e usados para o benefício dos resultados no processo ensino-aprendizagem. Porém, [Pereira et al. 2017] apontaram que a variedade e a diversidade das fontes de dados possíveis de se trabalhar, obtidas de diferentes interfaces e contextos, traz problemas de interoperabilidade semântica, propriedade e privacidade dos dados.

Diversos dados são importantes para geração de informação em LA. Na prática, as IES possuem diferentes bases de dados, como dados relacionais e dados textuais não estruturados, que possuem informações úteis, como a interação do estudante em fórum,

\footnotetext{
${ }^{1}$ https://moodle.org/

${ }^{2}$ https://www.sakaiproject.org/

${ }^{3}$ https://www.d21.com

${ }^{4}$ http://openredu.org/
} 
diálogo em chat em aprendizagem colaborativa, dados sobre modalidade de ensino, professor e conteúdo educacional.

O desafio que se busca solucionar com esta pesquisa é possibilitar com LA a geração de informações precisas, claras e úteis em tempo hábil aos estudantes e equipe pedagógica visando melhoria da tomada de decisão. A motivação para incluir Big Data na proposta de solução é a possibilidade de trabalhar com uma maior variedade e quantidade de dados, contemplando várias fases do processo educacional e buscando gerar modelos mais próximos da realidade.

O presente artigo está estruturado da seguinte forma: na Seção 2 são apresentados trabalhos relacionados a essa proposta; a Seção 3 detalha a proposta, apresentando os benefícios, motivações, colaborações e dificuldades para solucionar o desafio; a Seção 4 apresenta as discussões finais da proposta desse trabalho.

\section{Trabalhos Relacionados}

Kune et al. [R. Kune et al. 2016] fornecem uma visão geral dos sistemas de computação para Big Data, discutindo e diferenciando as principais camadas na arquitetura em relação ao modelo de banco de dados tradicional. Além disso, os autores identificam e classificam as lacunas na área de pesquisa em Big Data, afirmando que há necessidade de pesquisas em diversas áreas da computação ligadas ao assunto, como organização de dados, tomada de decisão, ferramentas específicas de domínio e ferramentas de plataforma. Isso pode criar uma infraestrutura de Big Data de próxima geração que permite aos usuários extrair o máximo benefício das grandes quantidades de dados disponíveis.

Rigo et al. [Rigo et al. 2012] mostram a relação de LA com dados, mineração de dados educacionais, ferramentas de avaliação de aprendizagem e trajetórias de aprendizagem, reforçando que o desenvolvimento de pesquisas em LA traz benefícios amplos para a comunidade de Informática na Educação (IE). Estes autores apontam também a possibilidade de obtenção de dados brutos e a atualização dos significados (semântica) dos dados, ao se fazer uma síntese sobre o processo LA.

A Revisão Sistemática da Literatura (RSL) realizada por [Krikun 2017] teve como objetivo especificar um processo, que inclui LA, capaz de melhorar a qualidade e a eficácia da aprendizagem em AVA's. A partir dos trabalhos levantados na revisão, foi observado o uso de LA e Mineração de Dados Educacionais (EDM) para as seguintes ações: classificação, e.g. classificar estudante em diferentes conjuntos; clustering, e.g. levantar grupos de estudantes que precisam do curso adaptado; regras da associação, e.g. descobrir relações entre elementos do curso usados pelo estudante; previsão, e.g. prever as dependências de usar o conteúdo e atividades; árvores de decisão, e.g. analisar aprendizagem.

Sistemas já conhecidos, e.g. Moodle, já possuem recursos mais simples de LA. De acordo com [Martins and Giraffa 2015], as técnicas de LA disponíveis no Moodle visam apoiar o trabalho do professor, auxiliar a avaliar os estudantes e facilitar a gestão dos materiais. Existem recursos como os relatórios sobre o progresso dos estudantes e alertas sobre atividades agendadas.

\section{Proposta}

$\mathrm{Na}$ atualidade, é crescente a utilização dos AVA's principalmente no que tange ao ensino superior. Esses ambientes possuem capacidade de coletar, armazenar e disponibili- 
zar grande quantidade de dados estruturados e não estruturados relativos à interação de estudantes e professores, oferece oportunidades de coleta de dados educacionais e estratégicos para realização de análises e avaliação monitoradas com o objetivo de garantir e aprimorar o processo de aprendizagem e o crescimento do estudante. Portanto, abordar tais questões pode proporcionar uma extensão e uma melhoria dos sistemas de apoio à decisão atualmente empregados pela gestão da educação, melhorando assim sua utilidade e aplicabilidade prática.

Para os referenciais de qualidade nos AVA's, o MEC afirma que o modelo de avaliação da aprendizagem precisa auxiliar o estudante a desenvolver competências e habilidades por meio de um processo contínuo, utilizando, para isso, uma verificação contínua do progresso dos estudantes por meio de atividades à distância e também avaliações presenciais. Desse modo, devem ser articulados mecanismos que promovam o permanente acompanhamento dos estudantes no intuito de identificar eventuais dificuldades na aprendizagem e saná-las ainda durante o processo de ensino-aprendizagem [Secretaria de Educação a Distância, MEC 2007]. Este cenário motivou a proposta de uma abordagem capaz de trabalhar LA e dados de variadas fontes, possibilitada pela adoção de Big Data, conforme a arquitetura ilustrada na Figura 1.

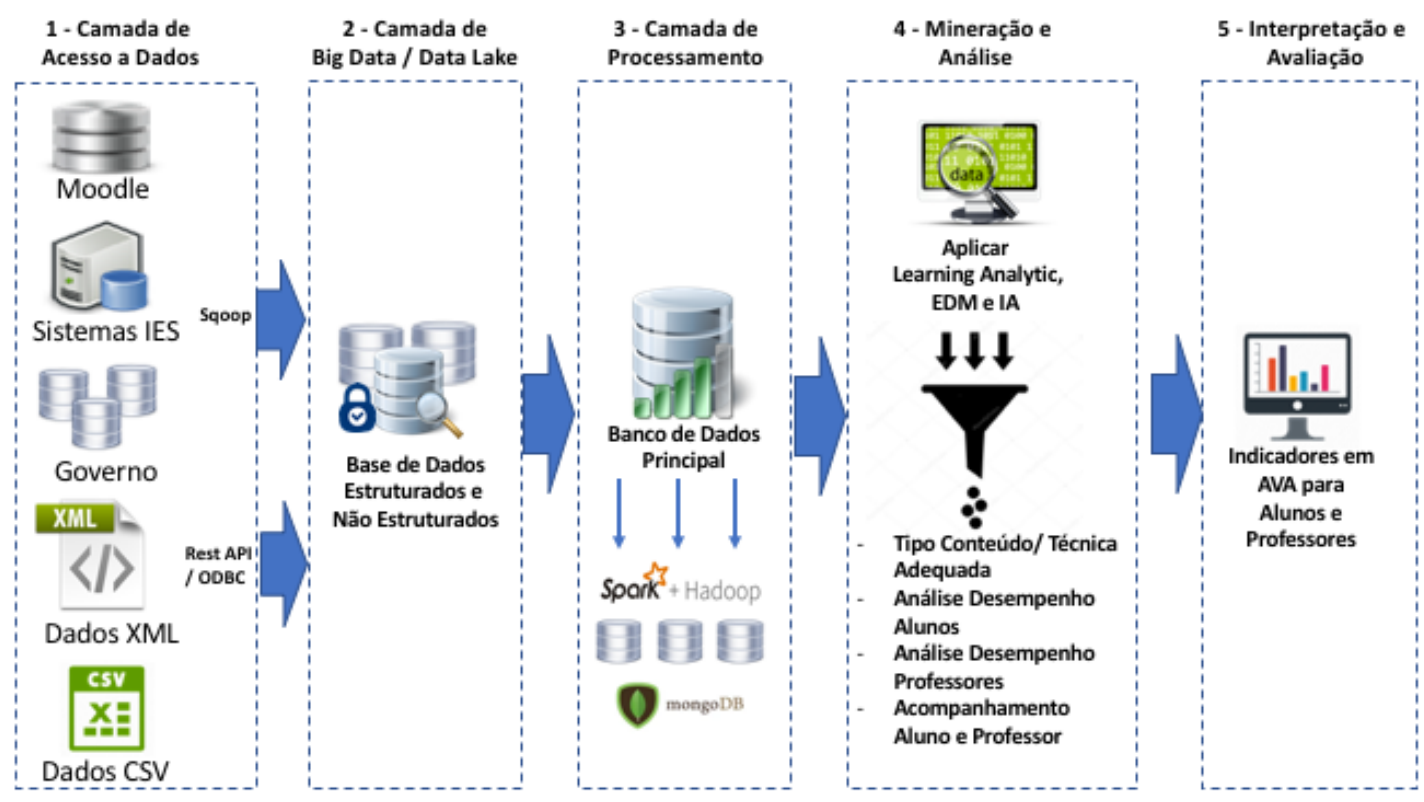

Figura 1. Proposta de Arquitetura

Fonte: baseado em [Alblawi and Alhamed 2017]

1. A camada de acesso a dados deverá ser responsável pelos acessos aos bancos de dados existentes como os da plataforma Moodle, bases de sistemas acadêmicos, arquivos de sistemas governamentais e arquivos em formato Comma-Separated Values (CSV) ou Xtensible Markup Language (XML); para o trabalho deverá ser utilizada tecnologias tais como Sqoop, Rest API, Open Database Connectivity (ODBC) para as conexões apropriadas aos bancos de dados. Para o caso de novas bases de dados, estas deverão utilizar uma das tecnologias existentes na arquitetura 
proposta de conexão ou exportar os dados nos formatos de CSV ou XML para realizar a importação para o Big Data;

2. A camada de Big Data / Data Lake inclui os repositórios dos dados coletados de diversos bancos e arquivos. Em virtude da tecnologia do Big Data possibilitar o agrupamento de grande quantidade de dados, oriundas de diversas fontes, diferentes formatos e estruturas irá favorecer ao trabalho e justifica o uso desta tecnologia. Este novo banco a ser alimentado deverá armazenar dados educacionais como o perfil do estudante e professores, registro acadêmico ou código dos estudantes e professores, disciplinas ministradas, atividades aplicadas, notas recebidas, médias de aprovações, tipo de atividades aplicadas, feedback realizados, informações advindas de chats, fóruns utilizadas nos ambientes de ensino das IES e que constam nestas bases a serem importadas e analisadas. É importante salientar que quaisquer dados que possam identificar os estudantes, professores e tutores deverão ser criptografados, afim de se manter o anonimato destes entes.

3. A camada de processamento será responsável por processar em paralelo o grande volume de dados do Big Data, para então possibilitar a geração novas bases já consolidadas. Para o correto processamento de grandes volumes de dados será necessária a adoção de novas ferramentas e técnicas especiais para a eficiente e correta manipulação, motivando a utilização de novas tecnologias como Hadoop, Spark em conjunto com a base de dados NoSQL mongoDB. Tecnologias essas já conceituadas e amplamente utilizadas no mercado, sendo todas de licença livre criadas pela fundação Apache Software Foundation (ASF). A escolha se deu devido a ausência de custos financeiros, a capacidade de processamento em paralelo, escalabilidade e ainda proporcionar cálculos rápidos com alta eficiência na manipulação de dados no Big Data;

4. Já na camada mineração e análise pretende-se aplicar técnicas de análise e predição em LA como Social Network Analysis(SNA), Sentiment Analysis, Influence Analytics, Learner Success Prediction, já em EDM deverá ser utilizado técnicas como classification, Relationship Mining, em conjunto com técnicas de IA como Bayesian Modeling, com o intuito de extrair informações no processo ensinoaprendizagem educacional existentes nas bases de dados. O uso das técnicas de LA, EDM em conjunto com IA se justifica pela possibilidade de buscar e localizar informações estratégicas para os professores, a fim de compreender e otimizar a aprendizagem, dentro do processo de ensino-aprendizagem.

5. A camada de interpretação e avaliação dedica-se a reportar as informações através de painéis de dashboard e relatórios gráficos com informações das evoluções dos estudantes e professores de forma segura e individual. Para os estudantes, espera-se mostrar em forma de gráficos os dados analíticos e detalhados de desempenho tais como total de horas no ambiente, interações com estudantes e professores, publicações feitas, conversas realizadas em chats, fóruns, percentual de atividades completadas, notas e faltas recebidas nas disciplinas e quais foram as práticas e materiais adotados que possibilitam esses resultados e com isso detectar e predizer risco de falhas. Aos professores, espera-se apresentar informações como padrões de comportamentos de estudantes nos ambientes virtuais, resultados de evoluções nas disciplinas por estudante, feedback, possibilitando com isso criar uma correta análise para o acompanhamento individual dos estudantes. Esperase ,ainda, trazer aos professores análises e sugestões para melhorar o processo 
ensino-aprendizagem nos ambientes AVA's, proporcionando predizer e detectar risco de falhas, bem como formas de intervenções para facilitar o progresso e aprendizado dos estudantes.

A arquitetura proposta poderá ser utilizada como uma possível solução para a chamada ao desafio proposto pelo $7^{\circ}$ DesafIE, pois acredita-se que ao se buscar novas bases de dados de ensino superior oriundas de diversas instituições para se montar o Big Data, estaremos contribuindo para a unificação e formação de um novo e grande repositório de dados, que deverá ser utilizado pelas IES, possibilitando extrair informações cujos custos são elevados para obtenção.

Após a coleta dos dados, estes deverão ser reestruturados e processados para a correta leitura e aplicação das técnicas de LA, EDM e IA, possibilitando a extração e exibição dos dados estratégicos de estudantes e professores em ambientes AVA's. A aplicação destas técnicas se faz necessária ainda que estas informações já existam nas bases, pois podem ser ignoradas ou não há agilidade na extração para a detecção de problemas no processo de ensino-aprendizagem. A deficiência é observada por desconsiderar grande parte dos dados que são coletados nos ambientes de aprendizagem, ou, em alguns casos, a forma incorreta de tratamento destes dados.

Pretende-se ,ainda, com o resultado deste trabalho ajudar as IES a monitorar e diagnosticar de forma objetiva, auxiliando a extrair algumas informações, tais como: o tipo adequado de conteúdo para aprimorar o aprendizado, o correto acompanhamento dos estudantes e de professores na aplicação de estratégias educacionais em ambientes online, predição de desempenho da aprendizagem de estudantes com dificuldade de aprendizagem, auxiliar e detectar possíveis problemas com antecedência para que possam ser providenciadas intervenções a fim de identificar os estudantes em risco de reprovar num curso. Contudo, não é o intuito deste trabalho substitui as diversas bases de dados atuais já existentes e devidamente estruturadas, pois todas as análises e dados obtidos deverão ser retornados às IES e demais órgãos governamentais para as devidas aplicações em seus ambientes.

\subsection{Benefícios e motivações para solucionar o desafio}

O uso crescente de AVA como ferramenta auxiliar no processo de ensino-aprendizagem proporciona uma dinâmica e modernização na educação, pois a utilização desses ambientes permite a disponibilização de materiais, controle de frequência, uso de chats, fóruns, entre outros. Porém, no contexto de AVA's, um dos desafios atuais está diretamente ligado com o tratamento de seus dados.

O desafio inicial consiste na seleção de dados de diferentes fontes de dados no intuito de conseguir aplicar as técnicas de LA para gerar informações úteis aos envolvidos no processo ensino-aprendizagem. A proposta deste trabalho visa dar suporte a partir desta etapa, onde a coleta de dados é o primeiro desafio. Tendo a oportunidade de coletar os dados de forma correta, informações dinâmicas devem ser geradas sobre o estado em que se encontra a aprendizagem individual e coletiva.

Ainda é notada uma deficiência com relação ao tipo de feedback dado aos envolvidos no processo educacional. Neste aspecto, isto deve ser caracterizado como um desafio. As informações cruas podem não ser tão eficientes se comparadas com visualizações dinâmicas. LA associada a técnicas de visualização da informação ou Interação 
Humano-Computador (IHC) traz grandes valores a professores, estudantes e administradores, possibilitando feedback sobre envolvimento, previsão de desempenho e evasão de estudantes [Junior and Oliveira 2016]. Assim, observa-se que outro benefício desta proposta é o uso de gráficos e imagens como estratégia facilitadora na visualização de informações geradas a partir de dados coletados.

\subsection{Colaborações para solucionar o desafio}

Apesar das iniciativas do governo brasileiro sobre dados abertos, ainda há questões políticas que influenciam na obtenção de dados de sistemas de IES brasileiras, sejam estes dados de sistemas acadêmicos ou oriundos de AVAs. A obtenção desses dados depende, entre outros, de aprovação dos projetos de pesquisa pelos comitês de ética das IES e também de um trabalho de tratamento dos dados para garantir a privacidade dos indivíduos.

Levantamento feito em 2014 e 2015 por [Junior and Oliveira 2016], houve um alto número de trabalhos publicados na Europa, com representativa participação da Espanha, e o continente Americano, representado pelos Estados Unidos, totalizando 48\% e 32\% dos trabalhos analisados. É possível perceber que há pesquisadores em diferentes localizações interessados e trabalhando o tema.

LA tem capacidade de gerar informações importantes just in time (no momento adequado) para ser usada nas IES de maneira continuada, afim de melhorar seu processo ensino-aprendizagem. Diante disso, é proposta uma colaboração entre pesquisadores de IE e gestores das IES, buscando, de maneira consciente, ambientes educacionais mais propícios para a pesquisa e desenvolvimento.

\subsection{Dificuldades atuais e vindouras ligadas ao desafio}

De acordo com [Junior and Oliveira 2016], os trabalhos sobre o tema apresentaram em sua maioria dificuldades relacionadas aos dados que revelaram a necessidade de propor mecanismos para pré-processar os dados do AVA, contextualizar e dar significado aos mesmos, além de trabalhar com dados abertos. Ficou evidenciado a necessidade de que haja interoperabilidade entre ferramentas de LA e de e-Learning, o que traz a necessidade de propor uma arquitetura, considerando que diferentes tecnologias podem ser usadas como componentes, tal qual permita a integração entre as ferramentas, como os AVA's, as ferramentas de análise de dados e tecnologias semânticas.

Apesar das Instituições de Ensino possuírem grandes quantidades de dados, o uso desses dados para a monitoração, predição, mitigação da evasão escolar tem sido ineficiente [Rigo et al. 2012]. como:

Algumas dificuldades, atuais e vindouras, podem ser destacadas e resumidas

- Acesso aos dados: Dificuldade de acesso aos dados em instituições públicas e particulares: fontes diversas, nem tudo está em ambiente informatizado (muitos processos manuais), restrições políticas e burocráticas.

- Confiabilidade dos dados: alguns questionamentos podem ser feitos em relação aos dados, tais como, "O dado equivale à realidade?", "O estudante colou?A sequência de atividades feitas tem uma linha de continuidade de acerto/erro?". A garantia da confiabilidade dos dados é um desafio que não deve ser ignorado. 
- Saneamento dos dados/Curadoria: dificuldade em ter dados saneados para conseguir efetividade na análise de LA. A etapa de pré-processamento dos dados é essencial para eliminar dados redundantes e inconsistentes e, tal processo determina a eficiência dos algoritmos utilizados em LA.

- Dificuldade em utilizar os informações: dificuldade de obter informações relevantes para uso no processo ensino-aprendizagem no momento correto ("just-intime").

- Integração entre plataformas: dificuldades em se integrar com diferentes fontes de dados e de disponibilizar os resultados em diversos AVA; dificuldade de encontrar plataformas abertas com possibilidade de integração com LA, e.g. atualmente muitos trabalham com o Moodle, que traz dificuldades de integração.

Outro desafio que merece destaque é como validar a efetividade da melhoria da qualidade das IES e consequentemente, dos cursos, estudantes e professores. Pois, além dos aspectos computacionais, como coleta dos dados e geração de informação, é necessário que os tomadores de decisão consigam compreender os indicadores apresentados. Portanto, é necessário despertar o interesse de todos os envolvidos no processo ensinoaprendizagem para que colaborem na geração de dados, bem como no uso das informações.

\subsection{Como enfrentar o desafio no período de 10 anos}

Como observado nas subseções anteriores, existe um desafio com relação ao trabalho de LA e a solução deve vir através de parcerias para que dados e ferramentas estejam disponíveis, permitindo que o trabalho colaborativo seja viável. Assim, algumas etapas estão sendo propostas.

A primeira etapa consiste em organizar recursos e adquirir conhecimentos suficientes, considerando os aspectos de pesquisa aplicada, para o desenvolvimento do tema. Estima-se 2 anos o tempo para execução desta etapa.

- Parcerias com IES: A necessidade de dados é fundamental para a execução do trabalho. Desta forma, o acesso a diferentes base de dados, por meio de parcerias com IES públicas e/ou privadas, deve trazer um volume de dados considerável para a primeira etapa. Parcerias com órgãos de Educação do Governo ligados às IES trazem grandes contribuições neste momento.

- Levantamento do Estado da Arte: É necessário um aprofundamento dos estudos que proporcionem a compreensão do estado da arte sobre LA, EDM e construção de Big Data agregando diferentes bases de dados educacionais. É necessário visualizar as características ligadas aos requisitos computacionais e à característica aplicada da pesquisa.

A segunda etapa consiste no desenvolvimento da ferramenta e 3 anos seriam necessários para a execução desta tarefa.

- Especificação e Desenvolvimento: Esta etapa deve consistir das subetapas a seguir:

- Criação das estruturas de dados para Big Data, capaz de lidar com dados estruturados e não estruturados;

- Especificação e implementação das interfaces para conexão com diferentes bases de dados; 
- Implementação das técnicas para extração, criptografia e padronização dos dados;

- Implementação das técnicas de LA, EDM e IA para extração de padrões e indicadores;

- Implementação da visualização da informação em LA, na forma de Dashboard's com indicadores e informações para estudantes, professores e gestores;

- Testes com a ferramenta;

- Validação: Realizar testes com a ferramenta em ambientes educacionais reais utilizando uma metodologia empírica e confiável. É necessário envolver IES e seus profissionais nesta atividade.

A terceira etapa consiste no envolvimento com comunidades acadêmicas. Estimam-se 4 anos o tempo para esta etapa.

- Publicação dos resultados: É esperado que os resultados da pesquisa sejam publicados para colaboração com a comunidade científica e acadêmica.

- Disponibilização da ferramenta: A partir do projeto implementado e validado, pretende-se disponibilizar às IES para utilização, possibilitando benefícios no diagnóstico e melhoria do processo ensino-aprendizagem.

- Envolvimento com a comunidade de IE: Realizar ações para viabilizar a associação de diferentes grupos de pesquisa ligados ao tema para a continuidade da pesquisa. Enquanto a proposta desta pesquisa envolve LA e Big Data, parcerias com grupos de pesquisa que trabalhem com temas correlatos, como AVA e aprendizagem colaborativa, podem trazer oportunidade de aperfeiçoar a especificação arquitetural visando adaptar-se a tais ferramentas.

\section{Discussões finais}

Apesar dos problemas elencados, o propósito deste trabalho é direcionar ações para solucioná-los, objetivando construir uma arquitetura baseada em LA e Big Data capaz de integrar com diferentes fontes de dados e gerar indicadores e informações para melhorar o processo ensino-aprendizagem das IES. Pretende-se, com parcerias em grupos de pesquisa da comunidade de IE, abordar aspectos computacionais e também aqueles relativos à aplicação, pois sabe-se das dificuldades ligadas ao custo de computação para Big Data e LA e das implicações dos fatores sócio-político-culturais relacionados à aplicação.

Espera-se que, nos próximos anos, o trabalho possa auxiliar as IES, professores e estudantes, no que tange a análise e extração de informações comportamentais e estratégicas. Com isso, prentende-se otimizar o tempo de aprendizagem e melhorar os resultados, proporcionando aos professores mais assertividade na aplicação dos conteúdos e avaliações, melhorando sua capacidade de tomar decisões e definir ações para motivar, orientar, acompanhar e avaliar os estudantes. Pois, sem o apoio tecnológico, seria muito difícil diagnosticar e melhorar o processo ensino-aprendizagem.

Nesta mesma linha de objetivos, espera-se que para aos estudantes sejam possibilitadas informações que contribuam para sua autoaprendizagem e autoavaliação e, consequentemente, reduzir o número de evasão. Os estudantes teriam acesso a informações que o auxiliam a traçar seu plano de estudos baseado em seu objetivo de aprendizagem, 
além de permitir preencher lacunas que possam estar ligadas ao ensino convencional, por meio de indicações, correções e reajustes.

De forma geral, mas não limitado a apenas essas, as principais contribuições desse trabalho são: (1) incentivar o uso de ambientes híbridos de aprendizagem (blended learning ou b-learning); (2) plataformas educacionais mais completas e eficazes; (3) disposição de forma acessível e clara de informações que auxiliam na detecção de estudantes com déficits de aprendizagem; (4) IES e professores terão em just in time informações sobre os estudantes, qualidade de ensino e material pedagógico, permitindo dessa forma, tomadas de decisões mais rápidas e assertivas; (5) possibilidade de adaptação do conteúdo a partir de recomendações baseadas nos resultados obtidos das análises dos dados; (6) avaliação da qualidade das IES, corpo professores e estudantes, infraestrutura e recursos didáticos.

\section{Referências}

Alblawi, A. S. and Alhamed, A. A. (2017). Big data and learning analytics in higher education demystifying variety, acquisition, storage, nlp and analytics. pages 126127.

Chatti, M. A., Lukarov, V., Thüs, H., Muslim, A., Yousef, A., Wahid, U., Greven, C., Chakrabarti, A., and Schroeder, U. (2014). Learning analytics: Challenges and future research directions. eleed, 10(1).

Gomes, A., Abreu, J., Claudeivan, L., and Veloso, F. (2017). Ensino e aprendizagem com a rede social openredu. In Tecnologias em EaD: Métodos e Práticas, Capítulo 1, pages 7-29. UFPB.

Junior, C. B. S. and Oliveira, I. C. A. (2016). Learning analytics: Revisao da literatura e o estado da arte. Congresso Internacional ABE de educação a Distância, pages 6-7.

Krikun, I. (2017). Applying learning analytics methods to enhance learning quality and effectiveness in virtual learning environments. In 5th IEEE Workshop on Advances in Information, Electronic and Electrical Engineering (AIEEE), pages 1-6. IEEE.

Martins, L. and Giraffa, M. (2015). Recursos de learning analytics para compor indicadores auxiliares na avaliação dos estudantes. Revista de Educação a Distância-EmRede, pages $37-38$.

Pereira, C. K. P., Siqueira, S. W. S., Nunes, B. P. N., et al. (2017). Dados conectados na educação. In $6^{\circ}$ DesafIE - Congresso da Sociedade Brasileira de Computação-CSBC, pages $742-743$.

R. Kune, P. K., Konugurthi, A.and Agarwal, R. R. C., and Buyya, R. (2016). The anatomy of big data computing, software: Practice and experience. In Jornal of Software: Practice and Experience, volume 46, pages 79-105.

Rigo, S. J., Cazela, S. C., and Cambruzzi, W. (2012). Minerando dados educacionais com foco na evasão escolar: oportunidades, desafios e necessidades. DesafIE - Congresso da Sociedade Brasileira de Computação-CSBC, pages 168-177.

Secretaria de Educação a Distância, MEC (2007). Referenciais de qualidade para educação superior a distância. In Ministério da Educação, Secretaria de Educação a Distância., pages 16-17. 